\title{
Periostin Mediates TGF- $\beta$-Induced Epithelial Mesenchymal Transition in Prostate Cancer Cells
}

\author{
Qingfeng Hua Shijun Tong ${ }^{a}$ Xiaojun Zhao ${ }^{b}$ Weihong Ding ${ }^{a}$ Yuancheng Gou \\ Ke Xua Chuanyu Sun ${ }^{\mathrm{a}}$ Guowei Xia ${ }^{\mathrm{a}}$ \\ a'Department of Urology, Huashan Hospital, Fudan University, Shanghai, 'Shanghai Centre for Clinical \\ Laboratory, Shanghai, China
}

\section{Key Words}

Epithelial mesenchymal transition • Periostin • TGF- $\beta$ • Prostate cancer $\bullet$ Lentivirus

\begin{abstract}
Background: In our previous study, we found that periostin was upregulated in prostate cancer, and its expression could be modulated by TGF- $\beta$. TGF- $\beta$ could upregulate periostin expression in some cells, and both TGF- $\beta$ and periostin could induce epithelial mesenchymal transition (EMT). We aimed to study the effect of periostin in the process of TGF- $\beta$-induced EMT in prostate cancer cells. Methods: We constructed a lentivirus vector containing the periostin gene and transduced it into PC3 and DU145 cells. After confirming periostin overexpression by PCR and Western blotting, we used an MTT assay to establish a growth curve to measure cell proliferation. Additionally, we performed transwell and wound healing assays to measure cell invasion and migration, respectively. Lastly, we measured the expression of EMT associated factors using Western blot analysis to test the effect of periostin on EMT in prostate cancer cells. Results: PCR and Western blot analyses confirmed that periostin was upregulated after infection with the periostin lentiviral vector. Periostin overexpression promoted increased cell proliferation, invasion, and migration as measured by MTT, transwell, and wound healing assays, respectively. Western blot analysis illustrated that periostin overexpression increased the expression of EMT associated factors, and periostin overexpression activated Akt and GSK$3 \beta$, which could be inhibited using a PI3K inhibitor. Additionally, TGF- $\beta$ increased the levels of STAT3, Twist1 and periostin, while both STAT3 shRNA and Twist1 shRNA inhibited periostin expression. However, STAT3 shRNA also decreased Twist1 expression. Although reduction of STAT3, Twist1 or periostin levels with shRNA inhibited TGF- $\beta$-induced overexpression of EMT associated factors, periostin overexpression could reverse such inhibition by interfering with STAT3 and Twist1. Similarly, periostin overexpression also reversed inhibition of cell invasion induced by interference of STAT3 and Twist1. Conclusion: Our findings indicate that periostin is an important mediator of TGF- $\beta$-induced EMT and suggest that periostin is a potential therapeutic target for suppressing the metastatic progression of prostate cancer.
\end{abstract}

Q. Hu, S. Tong and X. Zhao contributed equally.

Copyright $(2015$ S. Karger AG, Basel

Chuanyu Sun and Guowei Xia

KARGER 125
Middle Wulumuqi Road 12, Shanghai (China)

Tel. +86-021-52887080, Fax +86-021-52888030

E-Mail zhugexianglong@163.com,E-Mail xiaguowei@fudan.edu.cn 


\section{Introduction}

Invasion and metastasis are crucial for the progression and prognosis of cancer. These processes are initiated when cancer cells detach from their primary location and invade the surrounding tissue. Common features involved in decreased adhesion and increased motility and invasiveness could result from a trans-differentiation process referred to as the epithelial mesenchymal transition (EMT) [1]. In vitro, the EMT process can be recognized by the characteristics of spindle shape and pseudopodia, as well as increased intercellular separation [1]. Moreover, increased motility and decreased cell adhesion also indicate a mesenchymal phenotype, which could be reflected by the upregulation of mesenchymal proteins such as N-cadherin, fibronectin, and periostin [1].

Periostin, also known as osteoblast-specific factor 2, belongs to the superfamily of TGF- $\beta$ inducible proteins, which promote integrin-dependent cell adhesion and motility [2]. Periostin-associated signalling pathways promote numerous processes, such as cell growth and survival, angiogenesis and resistance to hypoxia-induced cell death [3, 4]. Our previous study has also demonstrated that periostin is upregulated in prostate cancer cells, and silencing its expression could inhibit cell proliferation [5]. It has been shown that periostin can induce EMT via the activation of the PI3K/Akt pathway in prostate cancer cells $[6,7]$. Additionally, it has been reported that TGF- $\beta$ can modulate periostin expression in periodontal ligament and gingival fibroblasts [8]. However, similar studies in tumour cells are still lacking. Moreover, the transcription factor Twist1 can modulate bone morphogenetic protein (BMP)-2-induced periostin expression, and TGF- $\beta$ can upregulate Twist1 expression through STAT3 [9]. Whether TGF- $\beta$ can induce periostin expression through Twist1 in prostate cancer remains unclear. However, TGF- $\beta$ could initiate the EMT process through activation of receptor tyrosine kinase signalling [10]. Periostin might mediate TGF- $\beta$-induced EMT in prostate cancer cells; however, the detailed interactions between TGF- $\beta$, periostin and EMT remain unclear. In the present study, we aim to clarify the effect of periostin in TGF- $\beta$-induced EMT in prostate cancer cells using lentiviral expression systems to study this molecular mechanism.

\section{Materials and Methods}

\section{Cell lines}

The prostate cancer cells, PC3 and DU145 used were from the Type Culture Collection of the Chinese Academy of Science. HEK 293T cells from American Type Culture Collection (ATCC) were used for the preparation of lentiviral vectors.

Construction of POSTN overexpression vector

The pCMV6 Entry vector and pLVX-IRES-ZsGreen1 vector were combined and collected in the Sgf I and Not I restriction enzyme reaction system after $3 \mathrm{~h}$ at $37^{\circ} \mathrm{C}$. A human periostin (RC215156) cDNA ORF clone from OriGene Technologies was inserted into the vector, and the ligation reaction proceeded at $4^{\circ} \mathrm{C}$ overnight. The plasmid was transformed into DH5 $\alpha$ competent cells that were cultured in ampicillin selection at $37^{\circ} \mathrm{C}$ overnight.

Lentivirus production and infection into cells

Periostin cDNA (OriGene Technologies) was inserted into the pHBLV-IRES-ZsGreen-PGK-puro vector (Hanbio, Shanghai, China) and co-transfected into 293T cells with plasmids psPAX2 and pMD2G using LipoFiterTM (Hanbio, Shanghai, China). Lentivirus-containing supernatant was harvested $48 \mathrm{~h}$ after transfection and filtered through $0.22-\mu \mathrm{m}$ cellulose acetate filters (Millipore, USA). Recombinant lentiviruses were concentrated by ultracentrifugation ( $2 \mathrm{~h}$ at 50,000 $\times \mathrm{g})$.

For lentiviral infection, the culture medium was removed when the PC3 and DU145 cells were at $60 \%$ confluence. The cells were washed gently with PBS and treated with the virus-containing medium (MOI $=10)$ combined with Polybrene $(5 \mu \mathrm{g} / \mathrm{ml})$. After 24 hours, the culture medium was replaced with fresh 


\section{Cellular Physiology Cell Physiol Biochem 2015;36:799-809 \\ \begin{tabular}{l|l|l} 
DOI: 10.1159/000430139 & (C)15 S. Karger AG, Basel
\end{tabular} \\ \begin{tabular}{l|l|} 
and Biochemistry Published online: May 22, 2015 & www.karger.com/cpb \\
\hline
\end{tabular}}

Hu et al.: Epithelial Mesenchymal Transition in Prostate Cancer

medium. After 72 hours of transduction, puromycin was added to the medium at a concentration of $5 \mathrm{mg} /$ $\mathrm{ml}$ for stable cell line selection. The empty lentiviral vector, lenti-puromycin, was used as negative control. Stable cells overexpressing periostin were obtained after 3 weeks of antibiotic selection. After harvesting these stable cells, the expression level of periostin was determined by western blotting and real-time PCR.

Lentiviral particles of periostin shRNA, STAT3 shRNA, Twist1 shRNA and empty-control were purchased from Santa Cruz, and the transfection methods used were the same as above.

\section{Detection of objective protein by Western Blot}

Cells were washed twice with $1 \times$ PBS and were then combined with $100 \mu \mathrm{L}$ of RIPA lysis buffer containing $1 \%$ PMSF protease inhibitor. Cells were lysed at $4^{\circ} \mathrm{C}$ for $20 \mathrm{~min}$, and the solution was centrifuged at 12,000 rpm for $30 \mathrm{~min}$. The supernatant was collected for protein assay using the bicinchoninic acid (BCA) method. Proteins were denatured in protein loading buffer containing 2-mercaptoethanol and bromophenol blue at $95^{\circ} \mathrm{C}$ for $10 \mathrm{~min}$. Gel electrophoresis proceeded at $60 \mathrm{~V}$ in the stacking gel and $100 \mathrm{~V}$ in the running gel for a duration of $2 \mathrm{~h}$, and proteins were transferred to PVDF membranes at $15 \mathrm{~V}$ and 0.1 A for $1.5 \mathrm{~h}$. All primary antibodies were obtained from Santa Cruz, and the secondary antibody was from Cell Signaling.

\section{Quantitative reverse transcription PCR}

Harvested cells were centrifuged and washed 3 times, and $1 \mathrm{~mL}$ of cell suspension was removed and centrifuged in the last wash cycle. The precipitate was mixed with $1 \mathrm{~mL}$ of TRIzol (Invitrogen, USA). After standing at room temperature $\left(15-30^{\circ} \mathrm{C}\right)$ for $5 \mathrm{~min}, 0.2 \mathrm{~mL}$ of chloroform was added with a $15 \mathrm{~s}$ quick shake. The solution was centrifuged $\left(15 \mathrm{~min}\right.$, at $\left.2-8^{\circ} \mathrm{C}, 12,000 \times \mathrm{g}\right)$, and the upper colourless aqueous phase containing RNA $(400 \mu \mathrm{L})$ was mixed with $0.5 \mathrm{~mL}$ of isopropanol at room temperature for 5-10 min. RNA was acquired in the precipitate after ultracentrifugation $\left(10 \mathrm{~min}\right.$, at $\left.2-8^{\circ} \mathrm{C}, 12,000 \times \mathrm{g}\right)$. After washing with $75 \%$ ethanol and centrifugation $\left(5 \mathrm{~min}\right.$, at $2-8^{\circ} \mathrm{C}, 7,500 \times \mathrm{g}$ ), the RNA precipitate was dissolved by diethylpyrocarbonate and used to synthesize cDNA. To synthesize cDNA, a $12 \mu$ l solution containing $1 \mu \mathrm{g}$ of RNA, $1 \mu \mathrm{L}$ of Oligo(dT) ${ }_{18}$ Primer, and $8 \mu \mathrm{L}$ of RNase-free water was incubated at $65^{\circ} \mathrm{C}$ for 5 min and rapidly cooled on ice. Then, $4 \mu \mathrm{L}$ of $5 \times \mathrm{RT}$ buffer, $1 \mu \mathrm{L}$ of RNase inhibitor, $2 \mu \mathrm{L}$ of dNTP $(10 \mathrm{mM})$, and $1 \mu \mathrm{L}$ of reverse transcriptase were added to a final volume of $20 \mu \mathrm{L}$. This solution was incubated at $42^{\circ} \mathrm{C}$ for $60 \mathrm{~min}$ and at $70^{\circ} \mathrm{C}$ for $10 \mathrm{~min}$. The synthesized cDNA could be used in PCR. The following primers for PCR were purchased from Invitrogen: POSTN-F, 5'-GAGCTTTACAACGGGCAAATAC-3', POSTN-R, 5'-CTCCCTTGCTTACTCCCTTTC-3', $\beta$-actin-F, 5'-CACTCTTCCAGCCTTCCTTC-3' and $\beta$-actin-R, 5'-GTACAGGTCTTTGCGGATGT-3'. The annealing temperatures for POSTN and $\beta$-actin were $55^{\circ} \mathrm{C}$ and $60^{\circ} \mathrm{C}$, respectively, and the reaction condition was $94^{\circ} \mathrm{C}$ for $30 \mathrm{~s}$, annealing temperature for $30 \mathrm{~s}$ and $72^{\circ} \mathrm{C}$ for $30 \mathrm{~s}$ in a total of 40 cycles. The PCR reactions consisted of $0.5 \mu \mathrm{L}$ of cDNA, $0.5 \mu \mathrm{L}$ of upstream primer, $0.5 \mu \mathrm{L}$ of downstream primer, $12.5 \mu \mathrm{L}$ of SYBR green supermix (TIANGEN, Beijing, China) and $11 \mu \mathrm{L}$ of deionized water. The $\Delta \Delta \mathrm{CT}$ method, calculated using ABI Prism 7300 SDS Software (ABI, USA), was used to analyze the expression.

\section{Measurement of cell growth curves and detection of cell invasion and migration}

The cell density was $2 \times 10^{4} / \mathrm{mL}$, and $200 \mu \mathrm{L}$ of MTT reagent was added to each well in a 96 -well plate. Beginning one day later, the MTT signal was measured for 5 days while the OD value was set at $570 \mathrm{~nm}$, and cell growth curves were constructed according to the mean OD values.

A transwell assay was used to detect the invasive capacity of cells in vitro, and the Transwell Insert (24-well Insert; pore size, $8 \mu \mathrm{m}$; Corning, USA) coated with ECM (extra-cellular matrix) gel (Sigma, USA) was employed. A total of $1 \times 10^{5}$ cells suspended in $0.1 \mathrm{ml}$ of fresh medium without fetal bovine serum were added to the insert. The cells on the upper surface of the membrane were removed using cotton buds after $48 \mathrm{~h}$. Cells that had migrated to the lower surface of the insert were fixed and stained with hematoxylin. Random visual fields of each insert were counted using a microscope $(200 \times)$.

A wound healing experiment was used to measure cell migration. A total of $1 \times 10^{6}$ cells were cultured on 6-well plates. A P-200 pipette tip (Qiagen, Valencia, CA) was used to scratch the cell layer upon confluence, and the cells were grown under normal conditions after being washed with culture medium. Photographs of the wound adjacent to reference lines scraped on the bottom of the plate were taken using microscopy $(100 \times)$, and wound healing was measured at 0 and 24 hours. Each experiment was completed three times. 


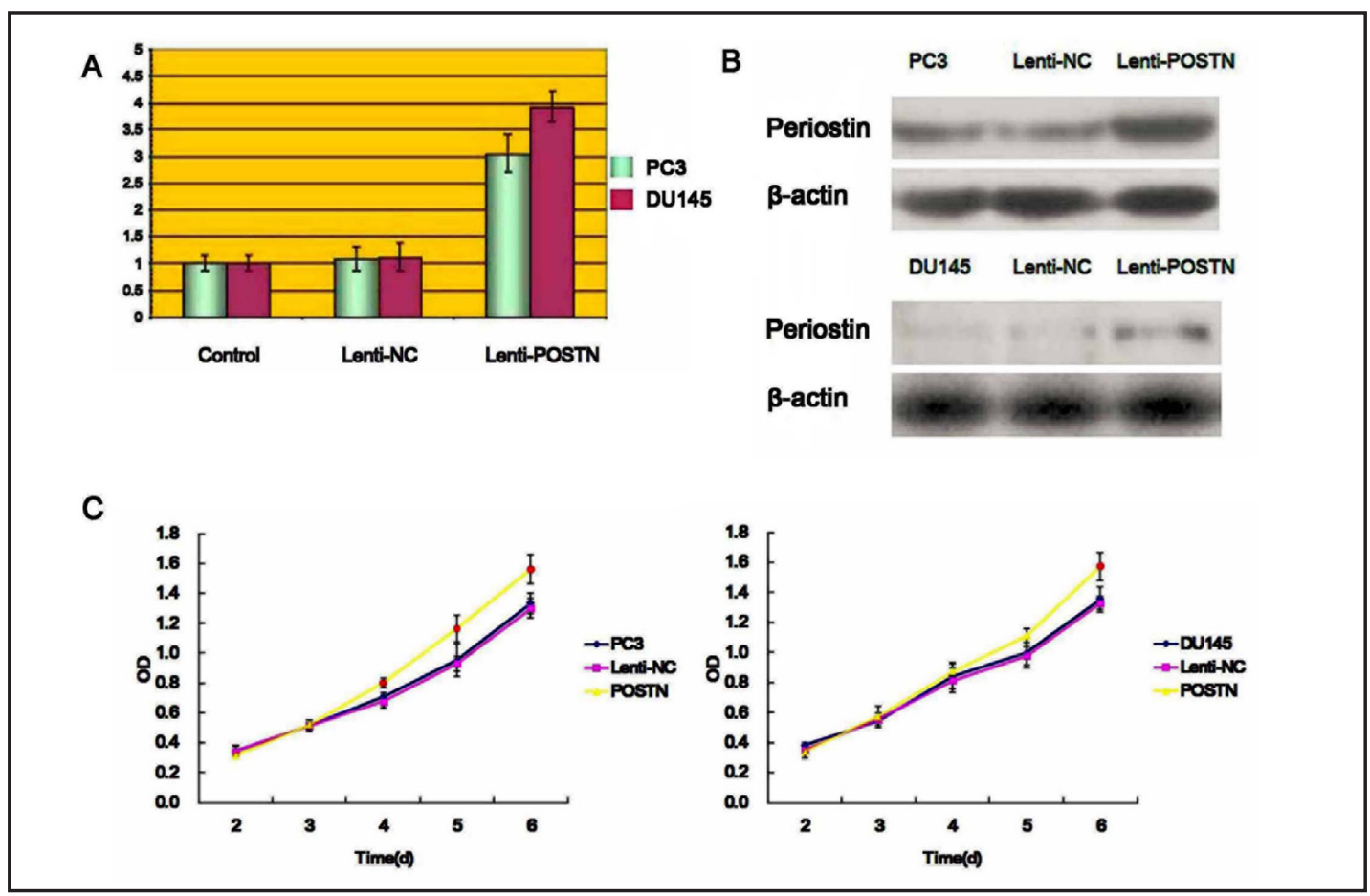

Fig. 1. The effect of periostin overexpression on cell proliferation in prostate cancer cells. (A) Quantitative reverse transcription PCR of PC3 and DU145 cells showed that the expression level of periostin were significantly higher that of controls. (B) Western Blot showed that lentivirus vector induced periostin overexpression compared to the controls. (C) Cell growth curves according to MTT test showed significantly increased cell proliferation induced by periostin. Elevated OD values in lentivirus transfected cells could be detected at the 4th (PC3 cells) and 6th (DU145 cells) day of culture, which was marked by red dots in the yellow lines showing cell proliferation of lentivirus transfected cells. NC: normal/empty control.

\section{Statistical analysis}

Data are presented as the mean $\pm \mathrm{SD}$, and statistical significance was determined using Student's $t$ test to compare two groups or an analysis of variance (ANOVA) with a Bonferroni post hoc test to compare three or more groups. All statistical analyses were performed using Statistical Product and Service Solutions (version 16.0; SPSS, Inc., Chicago, IL, USA). Differences were considered statistically significant when the $p$ value $<0.05$.

\section{Results}

The effect of periostin on cell proliferation, invasion and migration

Overexpression of periostin in lentivirus infected cells and the effect of periostin overexpression on cell proliferation. Quantitative reverse transcription PCR showed that the expression levels of periostin in PC3 and DU145 cells infected with the periostin lentivirus vector were significantly higher than those in the control group and empty-vector group ( $p$ $<0.001$ ) (Fig. 1A). Similar results were also found using Western blotting, lentivirus-induced periostin overexpression compared with the control groups could be detected in both PC3 and DU145 cells (Fig. 1B). Cell growth curves generated using an MTT assay were used to identify the effect of periostin on cell proliferation. Periostin induced an increase in cell growth and prolonged culture duration (Fig. 1C). Elevated OD values in the periostin group were observed by the 4th (PC3 cells) and 6th (DU145 cells) days, which indicated increased cell proliferation activated by periostin $(\mathrm{p}=0.0102$ and 0.0146$)$. 


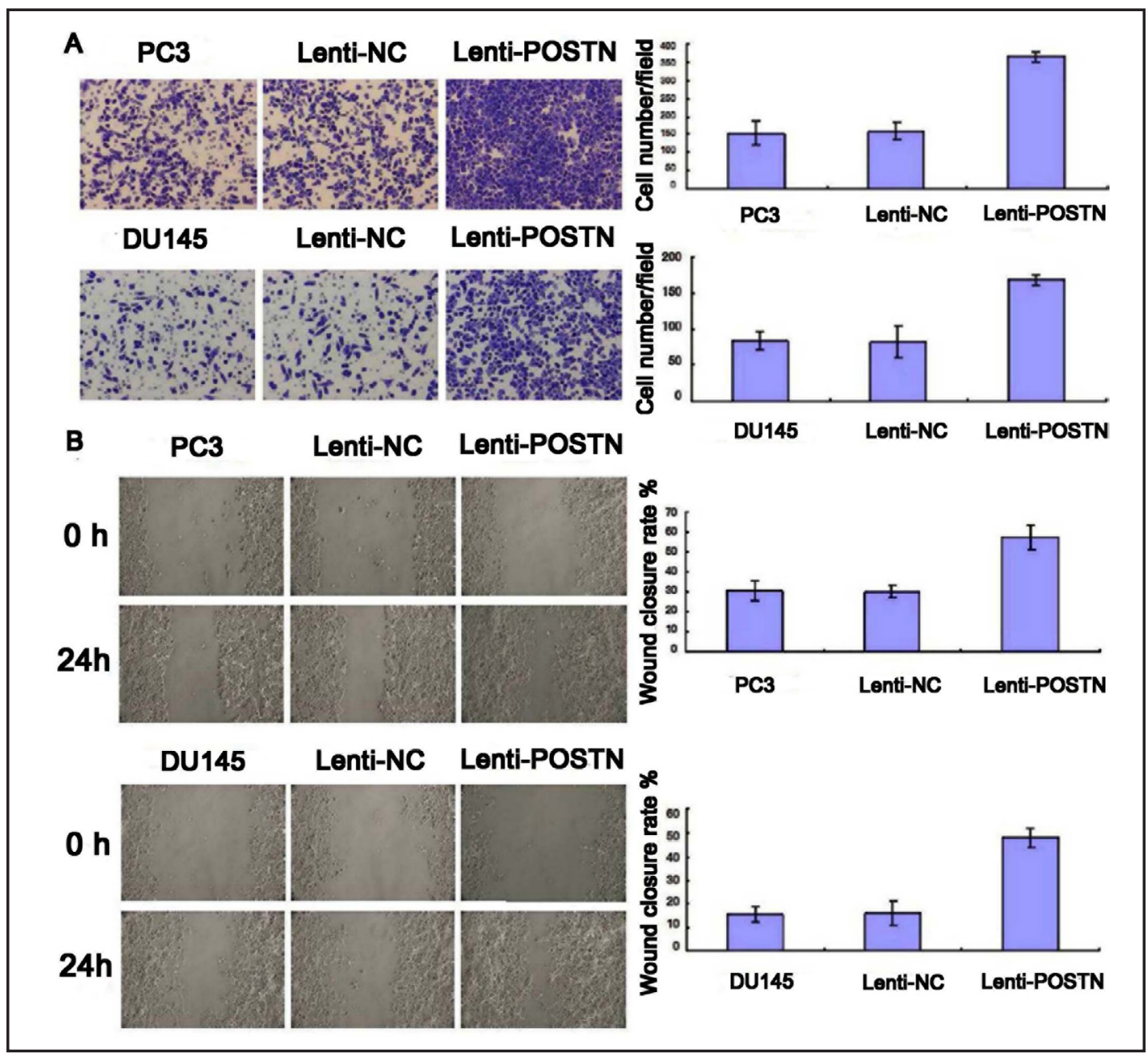

Fig. 2. The effect of periostin overexpression on invasion and migration. (A) Transwell assay showed that increased density of cells could be found in the periostin groups compared to the controls, indicating enhanced ability of cell invasion. (B) Wound healing experiments showed that raised wound closure rate in the periostin groups compared to the controls, indicating enhanced ability of cell migration.

The effect of periostin overexpression on invasion and migration. We found no significant difference in the total number of cells seeded into transwell chambers in each group after 48 hours ( $p>0.05)$. However, cell density on transwell inserts was increased in the periostin group compared with the normal and empty vector control groups, indicating an enhanced invasive capacity in periostin cells (Fig. 2A). There were more than twice as many invasive cells in the periostin group compared with the control groups in both PC3 (365 vs 152 vs 159 , $\mathrm{p}<0.001$ ) and DU145 (169 vs 83 vs $82, \mathrm{p}<0.001$ ) cells. The wound healing assay revealed an increased rate of wound closure in the periostin groups compared with the normal and empty vector control groups in both PC3 cells $(57.2 \%$ vs $30.6 \%$ vs $30.0 \%$, p < 0.001$)$ and DU145 cells (48.2\% vs $15.6 \%$ vs $16.1 \%$, p < 0.001$)$, indicating an enhanced cell migration ability (Fig. 2B). These results suggest that periostin overexpression can induce cell invasion and migration in prostate cancer cells.

The effect of periostin overexpression on EMT and associated PI3K/Akt signalling

The effect of periostin overexpression on EMT in prostate cancer cells. Western blot analysis showed significantly increased expression levels of the EMT-associated factors $\mathrm{N}$-cadherin and fibronectin in the periostin groups in both PC3 and DU145 cells. Although KARGER 


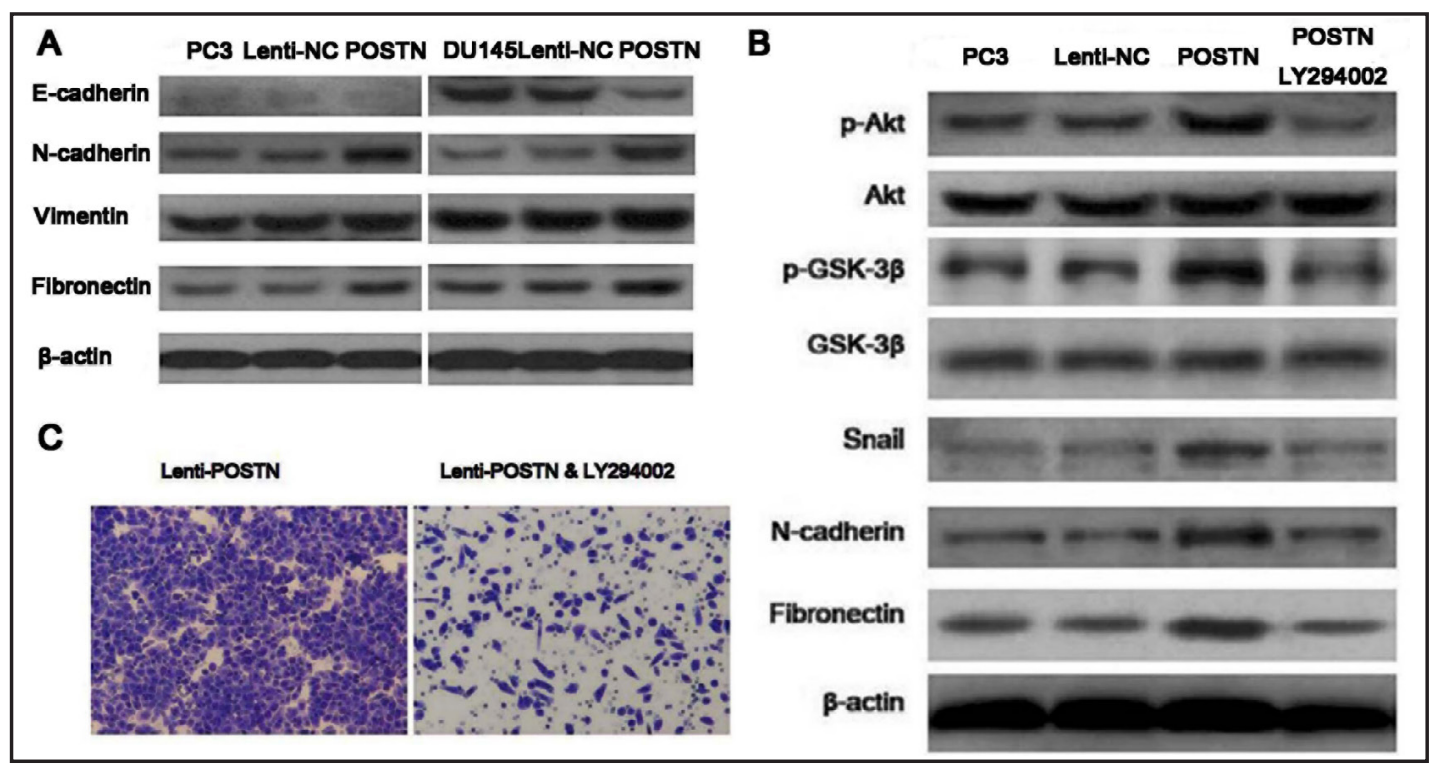

Fig. 3. The effect of periostin overexpression on EMT and associated PI3K/Akt signaling pathway. (A) Western Blot showed that periostin overexpression in PC3 and DU145 cells would increase the expression of $\mathrm{N}$-cadherin and fibronectin while inhibited the expression of E-cadherin, indicating that periostin overexpression could promote EMT in prostate cancer cells. (B) Western Blot showed that periostin overexpression would increase the expression levels of Akt, GSK-3 $\beta$, Snail, N-cadherin and fibronectin, which could be inhibited by PI3K/Akt signaling inhibitor LY294002. Periostin overexpression would induce the process of EMT via activation of PI3K/Akt signaling pathway. (C) The cell invasion due to EMT from periostin could be inhibited by PI3K/Akt inhibitor LY294002.

low expression of E-cadherin in PC3 cells might make a downregulation difficult to detect, significantly decreased expression of E-cadherin in the periostin group could be found in DU145 cells (Fig. 3A). Such results indicated that overexpression of periostin increased the expression of $\mathrm{N}$-cadherin and fibronectin and inhibited the expression of E-cadherin, indicating that periostin overexpression could promote EMT in prostate cancer cells.

Overexpression of periostin activating PI3K/Akt signalling pathway. We used PC3 cells transfected with a periostin lentiviral vector to test and verify the involvement of PI3K/ Akt signalling in the process of periostin-induced EMT. Western blotting indicated that periostin overexpression stimulated phosphorylation of Akt and GSK-3 $\beta$, and furthermore, increased expression levels of Snail, N-cadherin and fibronectin were detected, which could be inhibited by PI3K/Akt signalling inhibitor LY294002 (Fig. 3B). As mentioned previously, periostin overexpression induced the process of EMT via activation of PI3K/Akt signalling. Additionally, the increase in cell invasion by periostin-induced EMT was inhibited by LY294002 (Fig. 3C).

TGF- $\beta$-induced periostin expression and the effect of STAT3 and Twist1 on periostin expression

Dose- and time-dependent increases in periostin expression induced by TGF- $\beta$. To identify the effect of TGF- $\beta$ on periostin expression, we cultured PC3 cells in various TGF- $\beta$ concentrations for various durations (Fig. 4A). Periostin expression increased with increasing concentrations of TGF- $\beta$ after 48 h in PC3 cells. TGF- $\beta$ induced a dose-dependent increase in periostin expression after $48 \mathrm{~h}$ and a time-dependent increase in periostin expression at $10 \mathrm{ng} / \mathrm{ml}$ in PC3 cells. Additionally, periostin expression increased time-dependently with $10 \mathrm{ng} / \mathrm{ml}$ TGF- $\beta$ in PC3 cells. Together, these results indicated that TGF- $\beta$ induces periostin expression.

\section{KARGER}


Fig. 4. TGF- $\beta$ inducing periostin expression and the effect of STAT3 and Twist1 on periostin expression. (A) TGF- $\beta$ could increase periostin expression along with increased dose and time. (B) TGF- $\beta$ (10ng/ml and $48 \mathrm{~h}$ ) could induce overexpression of STAT3, Twist1 and periostin. The expression of Twist1 and periostin could be inhibited when treated with STAT3 shRNA and only the expression of periostin could be inhibited when treated with STAT3 shRNA, which indicated that STAT3 and Twist1 seemed to be mediators in the signaling pathway of TGF- $\beta$ induced periostin overexpression, and STAT3 was an upstream factor over Twist1 in this pathway.

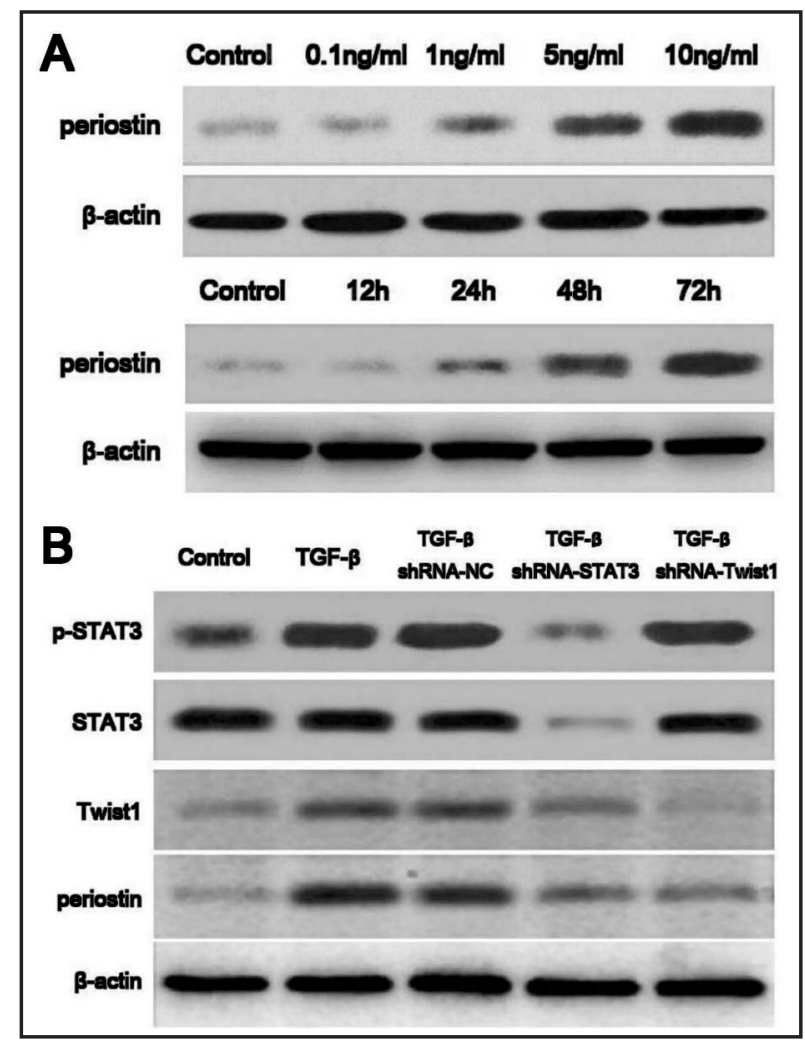

TGF- $\beta$ induces periostin expression through STAT3/Twist1 pathway. To verify the detailed effects of STAT3 and Twist1 on periostin expression, we used shRNAs targeting STAT3 and Twist1 to interfere with periostin overexpression in PC3 cells. All cells were cultured with 10 $\mathrm{ng} / \mathrm{ml} \mathrm{TGF-} \beta$ for $48 \mathrm{~h}$ and analysed by Western blotting. We found that the phosphorylation level of STAT3 was significantly elevated, and the protein levels of STAT3, Twist1 and periostin were increased when cells were treated with TGF- $\beta$. STAT3 shRNA inhibited STAT3 expression and phosphorylation and reduced both periostin and Twist 1 expression. However, when Twist1 expression was decreased by Twist1 shRNA, STAT3 expression was not significantly affected. As expected, we conclude that STAT3 and Twist1 appear to mediate the signalling pathway involved in TGF- $\beta$-induced periostin overexpression, and STAT3 is upstream to Twist1 in this pathway (Fig. 4B).

Periostin mediates TGF- $\beta$-induced EMT via STAT3/Twist1 signalling in prostate cancer cells

To verify the roles of STAT3, Twist1 and periostin in the process of EMT in prostate cancer cells, we used TGF- $\beta$ to induce EMT in PC3 cells and transfected shRNA to reduce EMT-associated factors to detect potential changes. Although Vimentin upregulation was weak, N-cadherin and fibronectin were upregulated significantly in PC3 cells treated with TGF- $\beta$, implying the presence of EMT. However, when we used periostin shRNA to decrease periostin expression, all EMT factors were downregulated, which indicates that TGF- $\beta$-induced EMT could be inhibited when periostin levels are reduced, and periostin is a downstream factor participating in the process of TGF- $\beta$-induced EMT (Fig. 5A). Additional results showed that either STAT3 shRNA or Twist1 shRNA could inhibit the upregulation of N-cadherin, vimentin and fibronectin, indicating that TGF- $\beta$-induced EMT could also be inhibited by interference with STAT3 or Twist1. However, this inhibition was blocked when periostin was overexpressed with the periostin lentiviral vector (Fig. 5B). Thus, periostin overexpression reversed the inhibition of EMT due to STAT3 and Twist1 shRNA, and the TGF- $\beta$ induced EMT process remained intact. These results indicated that STAT3/Twist1 act as upstream factors in TGF- $\beta$ /periostin signalling of EMT in prostate cancer cells.

\section{KARGER}




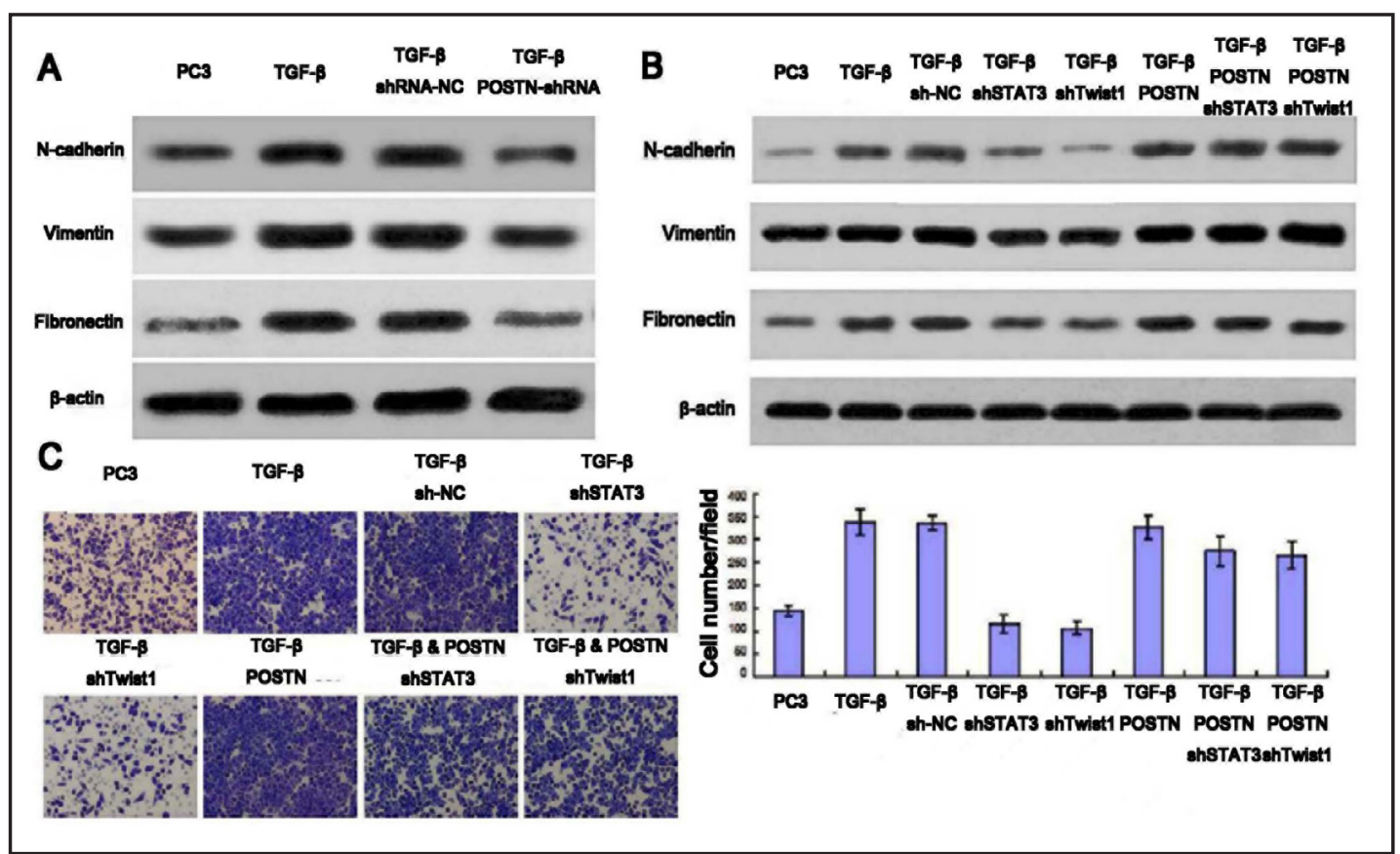

Fig. 5. Periostin mediating TGF- $\beta$-induced EMT via STAT3/Twist1 signaling pathway in prostate cancer cells. (A) Periostin downexpression due to periostin shRNA would induce N-cadherin, vimentin and fibronectin downexpression, indicating that perisotin should be a downstream factor participating in the process of TGF- $\beta$ induced EMT. (B) The inhibition of N-cadherin, vimentin and fibronectin by STAT3 shRNA and Twist1 shRNA could be reversed by periostin overexpression from periostin lentivirus vector. (C) The inhibition of cell invasion by STAT3 shRNA and Twist1 shRNA could be reversed by periostin overexpression from periostin lentivirus vector. These results indicated that STAT3/Twist1 should act as the upstream factors in TGF- $\beta$ /periostin signaling of EMT in prostate cancer cells.

This hypothesis was verified in the transwell assay (Fig. 5C). The average number of cells per field increased significantly when cells were treated with TGF- $\beta$ compared with untreated cells $(\mathrm{p}<0.001)$, which implied that TGF- $\beta$ enhanced the cellular invasion. Either STAT3 or Twist1 shRNA significantly decreased the numbers of invasive cells $(p<0.001)$ and inhibited the effect of TGF- $\beta$ on cell invasion. This inhibition was reversed when periostin was overexpressed using the periostin lentiviral vector and resulted in a rescue of TGF- $\beta$ induced cellular invasion in PC3 cells.

\section{Discussion}

We used lentivirus to construct a periostin overexpression vector in prostate cancer cells and found that periostin overexpression stimulated cell proliferation, invasion and migration. Furthermore, we confirmed the involvement of periostin in EMT in PC3 and DU145 cells and verified that periostin overexpression could activate PI3K/Akt signalling, as well as N-cadherin and fibronectin expression in PC3 cells. Additionally, periostin-induced cell invasion could be partially blocked by PI3K inhibitors. Together, these results confirmed that periostin activates EMT in prostate cancer cells via PI3K/Akt signalling. Furthermore, we found TGF- $\beta$ increased the levels of STAT3, Twist1 and periostin while STAT3 shRNA decreased Twist1 expression and both STAT3 shRNA and Twist1 shRNA together inhibited periostin expression, indicating that both STAT3 and Twist1 participated in the process of periostin upregulation induced by TGF- $\beta$ and that Twist 1 should be considered a downstream factor of STAT3. Finally, we found that periostin seemed to act as a downstream regulator of TGF- $\beta$-induced EMT in prostate cancer cells; reducing STAT3 or Twist1 levels inhibited TGF- 
$\beta$-induced EMT, and periostin overexpression reversed such inhibition. Similar results were also found in the transwell assay where reduction of STAT3 or Twist1 levels inhibited TGF$\beta$-induced cell invasion, which was reversed by periostin overexpression. Together, these results verified our hypothesis that periostin mediates TGF- $\beta$-induced EMT in prostate cancer cells. Additionally, STAT3/Twist1 signalling was involved between TGF- $\beta$ and periostin, and PI3K/Akt signalling should be considered a downstream pathway. To our knowledge, this is the first study to discuss the effect of periostin on EMT in prostate cancer cells and reveal a potential pathway of periostin participation in EMT induced by TGF- $\beta$.

EMT is accepted as a major event in the process of cancer cells acquiring the ability to invade and metastasize [11]. Numerous genes and signalling pathways have been reported to be involved in EMT, and the TGF- $\beta$ signalling pathway also plays an important role, which could initiate from the activation of Smad or non-Smad signalling pathways [12-14]. TGF- $\beta$ induced Smad signalling activates many transcription factors that mediate EMT, such as Snail and Twist. The activation of Snail transcription suppresses E-cadherin expression, which results in the acquisition of a mesenchymal phenotype and invasive behaviour in vitro [15, 16]. Yin et al. found that TGF- $\beta$ upregulated Twist1 in breast cancer cells, and the activation of Twist1 led to EMT and acquisition of breast cancer stem cell features [17]. In addition to the Smad signalling pathway, TGF- $\beta$ also promotes EMT through non-Smad pathways. Among these, PI3K/Akt signalling is a major pathway activated by TGF- $\beta$. TGF- $\beta$ activates PI3K/Akt/ mTOR, resulting in increased protein synthesis, motility and cell invasion [18]. Activation of the mTOR complex together with cytoskeletal reorganization leads to the transition of the epithelial cells to a mesenchymal phenotype [19]. TGF- $\beta$ induces a cellular morphological transition, smooth muscle actin expression and E-cadherin downregulation, and inhibitors of PI3K block the impact of TGF- $\beta$ on EMT $[20,21]$.

EMT of cancer cells could be characterized by the expression of hallmark proteins such as $\mathrm{N}$-cadherin, vimentin, and fibronectin, as well as periostin. Periostin has been reported to be involved in EMT of many cancer cells $[4,6,22,23]$ and is actively involved in the proliferation and migration of prostate cancer cells. We found that periostin was significantly upregulated in the biopsy tissue of prostate cancer patients compared with that of benign hyperplasia patients, and the numbers of migrated cells decreased with the decreased expression of periostin [5]. Moreover, periostin has been shown not only to be a marker of EMT but also to be an inducer of this process [24]. A previous study from Kim et al. demonstrated that the PI3K/Akt signalling pathway was involved in EMT induced by periostin in prostate cancer cells [6]. Periostin was able to induce EMT and promote increased invasion and migration by downregulating E-cadherin via Snail and increasing phosphorylation of Akt. We found that periostin overexpression in PC3 cells activated Akt phosphorylation and increased GSK-3 $\beta$, Snail, N-cadherin, and fibronectin expression, indicating that periostin should be considered as an important factor in activating PI3K/Akt signalling and inducing EMT in prostate cancer cells.

TGF- $\beta$ increased the expression of periostin, which has been previously shown in gingival and periodontal ligament fibroblasts [8]. However, the effect of TGF- $\beta$ on periostin expression in tumours has been seldom studied. Inai et al. have reported that Twist1 could modulate BMP- 2 to induce periostin expression, while other studies have reported that TGF- $\beta$ could upregulate Twist1 through STAT3 [9]. The defective link between TGF- $\beta$ /Twist1/ periostin and its effect on EMT in cancer cells becomes the key issue. Our study has verified that periostin induced EMT in prostate cancer cells through PI3K/Akt pathway similarly to what was mentioned by Kim's study [6]. Otherwise, Akt mediating TGF- $\beta$ induced EMT was also reported in the research of renal tubular epithelial cells recently [25]. Furthermore, we have found that TGF- $\beta$ induced periostin upregulation, while STAT3 and Twist 1 played a role that could not be ignored in the process. Moreover, periostin was also an important factor in TGF- $\beta$-induced EMT in prostate cancer cells. Therefore, a pathway in which TGF- $\beta$ could activate STAT3/Twist1 and upregulate periostin, further activating PI3K/Akt signalling and inducing EMT in prostate cancer cells, became clear.

\section{KARGER}


However, TGF- $\beta$-induced EMT is not limited to the Smad or non-Smad signalling pathways alone, and there is signalling crosstalk in EMT and other signalling such as micro RNA would also participate in this process [26]. Cho et al. found that the increased expression of Smad promoted the expression of Snail, which was required for the activation of Akt through the PI3K/Akt pathway in lens epithelial cells [27]. Similarly, in our study, we found that TGF- $\beta$ activated STAT3/Twist 1 and upregulated periostin to induce EMT via PI3K/Akt signalling in prostate cancer cells in a pathway in which Snail was also involved. These results could suggest that multiple signalling pathways are involved in the process of EMT induced by TGF- $\beta$, and these pathways cooperate together and contribute to TGF- $\beta$ induced EMT. The signalling of TGF- $\beta$ /STAT3/periostin/Akt could only be considered one of the potential pathways. As illustrated above, periostin is a key protein in the process of EMT. In clinical research, periostin expression was found to be increased both in stromal and epithelial tissues in advanced and metastatic tumours, indicating the important role of periostin in the process of cancer cells acquiring the ability to migration, invade and metastasize [28]. Actually, to inhibit EMT appears to be a promising therapeutic agent for tumours and other chronic diseases [29]. Our findings reveal that periostin has a major effect on EMT and is associated with increased cell migration, invasion and metastasis in prostate cancer. Further studies of periostin and its relationship with EMT could explain the mechanisms of metastasis in prostate cancer.

\section{Conclusions}

Periostin has attracted attention as an upregulated factor participating EMT in prostate cancer cells. We have revealed that periostin is induced by a TGF- $\beta$ /STAT3/Twist1 pathway and mediates TGF- $\beta$-induced EMT in prostate cancer cells. These findings indicate that periostin is an important mediator of TGF- $\beta$-induced EMT and suggest that periostin is a potential therapeutic target for suppressing the metastatic progression of prostate cancer.

\section{Disclosure Statement}

None.

\section{Acknowledgements}

This work was supported by the fund of NSF of China (81202033, 81372316) and ChenGuang Program of Shanghai Municipal Education Commission.

\section{Reference}

1 Thiery JP: Epithelial-mesenchymal transitions in tumour progression. Nat Rev Cancer 2001;2:442-454.

2 Takeshita S, Kikuno R, Tezuka K, Amann E: Osteoblastspecific factor 2: cloning of a putative bone adhesion protein with homology with the insect protein fasciclin I. Biochem J 1993;294:271-278.

3 Ouyang G, Liu M, Ruan K, Song G, Mao Y, Bao S: Upregulated expression of periostin by hypoxia in nonsmall-cell lung cancer cells promotes cell survival via the Akt/PKB pathway. Cancer Lett 2009;281:213219.

4 Li P, Oparil S, Feng W, Chen YF: Hypoxia-responsive growth factors upregulate periostin and osteopontin expression via distinct signaling pathways in rat pulmonary arterial smooth muscle cells. J Appl Physiol 2004;97:1550-1558; discussion 1549.

5 Sun C, Zhao X, Xu K, Gong J, Liu W, Ding W, Gou Y, Xia G, Ding Q: Periostin: a promising target of therapeutical intervention for prostate cancer. J Transl Med 2011;9:99.

6 Kim CJ, Sakamoto K, Tambe Y, Inoue H: Opposite regulation of epithelial-to-mesenchymal transition and cell invasiveness by periostin between prostate and bladder cancer cells. Int J Oncol 2011;38:1759-1766.

7 Baril P, Gangeswaran R, Mahon PC, Caulee K, Kocher HM, Harada T, Zhu M, Kalthoff H, Crnogorac-Jurcevic T, Lemoine NR: Periostin promotes invasiveness and resistance of pancreatic cancer cells to hypoxia-induced 
cell death: role of the beta4 integrin and the PI3k pathway. Oncogene 2007;26:2082-2094.

8 Wen W, Chau E, Jackson-Boeters L, Elliott C, Daley TD, Hamilton DW: TGF- $\beta 1$ and FAK regulate periostin expression in PDL fibroblasts. J Dent Res 2010;89:1439-1443.

9 Inai K, Norris RA, Hoffman S, Markwald RR, Sugi Y: BMP-2 induces cell migration and periostin expression during atrioventricular valvulogenesis. Dev Biol 2008;315:383-396.

10 Katsuno Y, Lamouille S, Derynck R: TGF- $\beta$ signaling and epithelial-mesenchymal transition in cancer progression. Curr Opin Oncol 2013;25:76-84.

11 Christiansen JJ, Rajasekaran AK: Reassessing epithelial to mesenchymal transition as a prerequisite for carcinoma invasion and metastasis. Cancer Res 2006;66:8319-8326.

$12 \mathrm{Xu}$ J, Lamouille S, Derynck R: TGF-beta-induced epithelial to mesenchymal transition. Cell Res 2009;19:156-172.

13 Moustakas A, Heldin CH: Signaling networks guiding epithelial-mesenchymal transitions during embryogenesis and cancer progression. Cancer Sci 2007;98:1512-1520.

14 Moustakas A, Heldin CH: Induction of epithelial-mesenchymal transition by transforming growth factor beta. Semin Cancer Biol 2012; 22: 446-454.

15 Hoot KE, Lighthall J, Han G, Lu SL, Li A, Ju W, Kulesz-Martin M, Bottinger E, Wang XJ: Keratinocyte-specific Smad2 ablation results in increased epithelial-mesenchymal transition during skin cancer formation and progression. J Clin Invest 2008;118:2722-2732.

16 Chen WC, Obrink B: Cell-cell contacts mediated by Ecadherin (uvomorulin) restrict invasive behavior of L-cells. J Cell Biol 1991;114:319-327.

17 Yin X, Wolford CC, Chang YS, McConoughey SJ, Ramsey SA, Aderem A, Hai T: ATF3, an adaptive-response gene, enhances TGF $\{$ beta\} signaling and cancer-initiating cell features in breast cancer cells. J Cell Sci 2010; 123: 3558-3565.

18 Lamouille S, Derynck R: Cell size and invasion in TGF-beta-induced epithelial to mesenchymal transition is regulated by activation of the mTOR pathway. J Cell Biol 2007;178:437-451.

19 Lamouille S, Connolly E, Smyth JW, Akhurst RJ, Derynck R: TGF- $\beta$-induced activation of mTOR complex 2 drives epithelial-mesenchymal transition and cell invasion. J Cell Sci 2012;125:1259-1273.

20 Bakin AV, Tomlinson AK, Bhowmick NA, Moses HL, Arteaga CL: Phosphatidylinositol 3-kinase function is required for transforming growth factor beta-mediated epithelial to mesenchymal transition and cell migration. J Biol Chem 2000;275:36803-36810.

21 Kattla JJ, Carew RM, Heljic M, Godson C, Brazil DP: Protein kinase B/Akt activity is involved in renal TGF-beta1-driven epithelial-mesenchymal transition in vitro and in vivo. Am J Physiol Renal Physiol 2008;295:F215-225.

22 Bao S, Ouyang G, Bai X, Huang Z, Ma C, Liu M, Shao R, Anderson RM, Rich JN, Wang XF: Periostin potently promotes metastatic growth of colon cancer by augmenting cell survival via the Akt/PKB pathway. Cancer Cell 2004;5:329-339.

23 Gillan L, Matei D, Fishman DA, Gerbin CS, Karlan BY, Chang DD: Periostin secreted by epithelial ovarian carcinoma is a ligand for alpha(V)beta(3) and alpha(V)beta(5) integrins and promotes cell motility. Cancer Res 2002;62:5358-5364.

24 Yan W, Shao R: Transduction of a mesenchyme-specific gene periostin into 293T cells induces cell invasive activity through epithelial-mesenchymal transformation. J Biol Chem 2006; 281: 19700-19708.

25 Lan A, Qi Y, Du J: Akt2 mediates TGF- 31 -induced epithelial to mesenchymal transition by deactivating GSK3 $\beta$ /snail signaling pathway in renal tubular epithelial cells. Cell Physiol Biochem 2014;34:368-382.

26 Teng Y, Zhao L, Zhang Y, Chen W, Li X: Id-1, a protein repressed by miR-29b, facilitates the TGF $\beta 1$-induced epithelial-mesenchymal transition in human ovarian cancer cells. Cell Physiol Biochem 2014;33:717-730.

27 Cho HJ, BaeKE, Saika S, Jeong MJ, Yoo J: Snail is required for transforming growth factor-beta-induced epithelial-mesenchymal transition by activating PI3 kinase/Akt signal pathway. Biochem Biophys Res Commun 2007;353:337-343.

28 Tischler V, Fritzsche FR, Wild PJ, Stephan C, Seifert HH, Riener MO, Hermanns T, Mortezavi A, Gerhardt J, Schraml P, Jung K, Moch H, Soltermann A, Kristiansen G: Periostin is up-regulated in high grade and high stage prostate cancer. BMC Cancer 2010;10:273.

29 Yang T, Chen M, Sun T: Simvastatin attenuates TGF- $\beta 1$-induced epithelial-mesenchymal transition in human alveolar epithelial cells. Cell Physiol Biochem 2013;31:863-874. 